\title{
PRÁCTICAS DE INTERCULTURALIDAD EN LOS PROCESOS EDUCATIVOS DE LA URACCAN, BILWI
}

Rosa Aura Palacios Rizo ${ }^{[1]}$ María Elena Watson Pérez ${ }^{[2]}$

\section{Resumen}

Cada docente a través de la mediación pedagógica en clase ha jugado un papel importante para el éxito escolar y el desarrollo de la interculturalidad, en atención fundamental a la diversidad étnica. Este estudio valoró las estrategias metodológicas implementadas en las clases en cuanto al desarrollo de la práctica de la interculturalidad en el primer año de Sociología, y, cuyos resultados se pretende sean valorados por la URACCAN para contribuir a la práctica de este eje transversal.

El enfoque fue el interaccionismo simbólico porque facilita describir aspectos educativos útiles para la práctica de la interculturalidad durante el proceso. Se realizaron entrevistas a estudiantes y docentes de la misma carrera y año, a la secretaria académica y a la coordinadora de Humanidades, asimismo se hicieron observaciones de algunas clases.

Entre los resultados es que algunos docentes redundan en trabajos de grupos y exposiciones, sin el complemento de una praxis de la interculturalidad que es un eje transversal en la URACCAN.

La interculturalidad en las clases representa una riqueza; pero, las estrategias metodológicas implementadas han tenido poca incidencia, por lo cual se requiere del diseño de estrategias metodológicas dentro del currículo de la carrera para contribuir al fortalecimiento de tales prácticas.

Palabras claves: Estrategias metodológicas, práctica de la interculturalidad, procesos educativos.

\footnotetext{
[1] Máster en Docencia Universitaria. rosapalacios62@yahoo.es

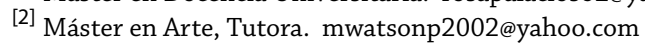




\section{Summary}

Each teacher through the classroom pedagogical mediation has played an important role for school success and development of interculturality, in primary attention to ethnic diversity. This study assessed the methodological strategies implemented in the classrooms related to developing the practice of interculturality in the first year of Sociology, and the results are intended to be valued by URACCAN in order to contribute to the practice of this transversal axis.

The focus was based on the symbolic interaction because it facilitates the description of educational aspects that are useful for the practice of interculturality during the process. Interviews were conducted with students and teachers of the same career and year, with the Academic Secretary and Coordinator of Humanities; also observations were made to some classes.

Among the findings it was demonstrated that some teachers are insistent with the group-works and presentations, without the complement of an intercultural practice that is a transversal axis in URACCAN.

Interculturality in the classrooms represents a treasure, but the methodological strategies implemented have had limited impact, so this requires the design of methodological strategies into the curriculum of the career to contribute to the strengthening of such practices.

Keywords: Methodological strategies, practice of interculturality, educational processes.

\section{Introducción}

El Informe de Desarrollo Humano 2005: Las Regiones Autónomas de la Costa Caribe, explica la conformación socio-étnica y cultural de los pueblos indígenas sumu/mayangna, miskitus, ramas, creoles, garífunas y comunidades mestizas que comparten un mismo territorio, como producto de la historia, economía, cultura, costumbres, religión y políticas donde cada pueblo construye sus memorias e identidades propias. Dentro de este contexto multicultural se requiere del enfoque e implementación de la Educación Intercultural Bilingüe como un derecho individual y colectivo de aspirar a la educación que se ofrece a toda la ciudadanía, asimismo mantener y cultivar sus propias tradiciones, cultura, valores, cosmovisión e idioma.

En este sentido, la URACCAN tiene la misión de contribuir al fortalecimiento del proceso de Autonomía de la Costa Caribe nicaragüense mediante la capacitación y profesionalización de recursos humanos, dotándolos de los conocimientos necesarios tanto para la conservación y el aprovechamiento de forma racional de los recursos 
naturales, cuyos objetivos estratégicos tienen como base cinco principios fundamentales: interculturalidad, autonomía regional, fortalecimiento institucional, excelencia académica y sostenimiento financiero.

La URACCAN como universidad comunitaria, brinda una formación cimentada en el principio de interculturalidad que permite a docentes y estudiantes, tener una relación horizontal con la gente de la comunidad, lo cual se afianza mediante espacios de interrelación, confianza y esperanza entre los sujetos multiétnicos. Sin embargo, aún se observan actitudes en el personal docente y estudiantes que no contribuyen al desarrollo de las prácticas interculturales durante el proceso educativo. Tal cual lo refirieren algunos estudiantes al observar que se redunda en la asignación de trabajos en grupos, dejando a libre opción la escogencia de los miembros de cada grupo, pero que laprincipal intención es desarrollar un programa de asignatura que se le ha encomendado.

\section{Revisión de literatura}

La URACCAN (2009:16), concibe la interculturalidad como un proceso donde se construyen un diálogo de iguales, donde las culturas pueden respetarse y aprender de las bondades de otras, crear juntos nuevos conocimientos en un marco de respeto y reconocimiento mutuo.

La interculturalidad como propuesta educativa desde el Sistema Educativo Autonómico Regional (SEAR; 2000,17), señala que: "promueve la valorización de las diversas culturas, estableciendo herramientas pedagógicas y científicas que contribuyan al diálogo, la articulación, la aceptación del otro u otra como legítima a través de relaciones respetuosas entre los pueblos indígenas y comunidades étnicas." Prácticas de interculturalidad.

Rizo (2003:26), describe la cultura como: "el conjunto de conocimientos, saberes, principios, técnicas, leyes y representaciones simbólicas que comparten los integrantes de un determinado pueblo. Sus costumbres, ordenamientos y creencias que los distinguen de los demás pueblos y culturas".

Ramírez (2002:14), manifiesta que la identidad cultural "es el proceso por el cual una persona se identifica", es decir, que se siente parte de una cultura y respeta, valora, participa de sus manifestaciones como ceremonias, ritos, costumbres, tradiciones. Estos son los principios en el constructo de currículum intercultural.

Arroyo (198:414), refiere que la enseñanza de la interculturalidad creativa: "es una modalidad didáctica que descubre ingeniosas formas de promover al aprendizaje y reacciona con sensibilidad ante las oportunidades que se presentan en el aula para despertar ese aprendizaje". 
Solá (1998:23), explica que estrategia: "es un conjunto planificado de acciones y técnicas que conducen al cumplimiento de objetivos preestablecidos en el proceso educativo".

Díaz \& Hernández (2005:45) señalan que: "las estrategias de enseñanza son procedimientos o recursos utilizados por el agente de enseñanza para promover aprendizajes significativos".

Según el Modelo pedagógico de la URACCAN (2004:16), la metodología a emplearse durante el proceso enseñanza - aprendizaje, debe ser: "Interactiva, que fomente la posibilidad de cambiar el papel, es decir, que el estudiante puede llegar a ser docente y el docente ser estudiante en la medida en que enfrentan nuevas situaciones de aprendizajes".

En este sentido, Rizo (2003:177) propone:

En las nuevas prácticas pedagógicas la escuela se puede adaptar a las culturas indígenas si la metodología de enseñanza se vuelve más flexible, donde se trata de valorizar el saber de los alumnos y alumnas e intercambiar con ellos experiencias y estudio.

Arroyo (1998: 414), precisa que: "en la enseñanza creativa intercultural, el maestro o maestra apoya de forma activa al alumno y alumna, lo cual se opone radicalmente a la transmisión del aprendizaje".

Refiere Kraine (1996: 44), que:: "para favorecer el proceso de interculturalidad, el maestro debe incorporar al aula los valores éticos y culturales de su propia comunidad, a partir de todos, introducir el diálogo con otras culturas"

Plantea Bolívar (1994:64), que un ámbito educativo intercultural amerita ser atendido con docentes, académica y profesionalmente bien formados y capacitados, capaces de respetar la individualidad e integridad de las personas y la cultura de las sociedades, dispuestos a favorecer la convivencia e interculturalidad de los pueblos y las personas.

Desde el modelo pedagógico de la URACCAN (2004:17), se considera a los estudiantes con capacidades y habilidades para desempeñarse como: “...Promotor de diálogo horizontal, respeto y promoción de relaciones equitativas de género y generacional. Orgullo de su pertenencia étnica. Comprometido con su formación y aprendizaje. Sentido de justicia, respeto y solidaridad". 


\section{Materiales y método}

Es un estudio cualitativo con enfoque en el interaccionismo simbólico, al abordar y describir aspectos sociales diferentes, útiles para la práctica y el fortalecimiento de la interculturalidad durante el proceso educativo en el primer año de la carrera de Sociología con mención en Autonomía. Se analizan las estrategias implementadas y su pertinencia en el desarrollo de la interculturalidad en clases.

El universo fue decuarenta (40) estudiantes, de los cuales, seis son varones y treinta y cuatro son mujeres. La muestra se tomó a diez estudiantes, seis docentes que impartieron clases en ese nivel. Se escogió por conveniencia, ya que la selección fue intencionada; es decir, que cada miembro de la muestra pertenecea una de las etnias que tienen presencia en la Costa Caribe nicaragüense y que cursan el primer año de la carrera de Sociología.

\section{Métodos y técnicas utilizadas}

Para la recopilación y obtención de datos se consideraron como fuentes primarias a docentes que facilitaron procesos educativos en el primer año de Sociología y a estudiantes activos del mismo nivel, a la coordinadora de la carrera de humanidades, asimismo la secretaria académica del Recinto Universitario Bilwi. Entre las fuentes secundarias se consideraron: revisiones bibliográficas afines al tema de estudio.

Se utilizaron como técnicas: entrevistas a docentes, estudiantes, secretaria académica y coordinadora de la carrera de humanidades, también observaciones a clases. Para el procesamiento de los datos se procedió al análisis, discusión e interpretación de los resultados de los instrumentos aplicados, posteriormente se procedió a la redacción del informe final.

\section{Aspectos éticos}

La información y datos obtenidos no serán utilizados para fines lucrativos sino que tienen el propósito que los resultados contribuyan para el desarrollo de las prácticas de interculturalidad durante el proceso educativo de estudiantes de la carrera de Sociología con mención en Autonomía y hacerlo extensivo a las demás carreras que oferta la URACCAN.

Además, los resultados serán compartidos con la coordinación del área de Humanidades. A fin de garantizar el desarrollo de la interculturalidad y la incidencia de la docencia, mediante las diversas estrategias metodológicas que implementan, en el deber de cumplir con la misión y visión de la universidad comunitaria. 


\section{Resultados y discusión}

\section{Estrategias metodológicas implementadas en el Recinto Bilwi}

La diversidad profesional y étnica del personal que se desempeñan como docentes en el primer año de la carrera de Sociología, son dos elementos importantes que permiten implementar variadas estrategias metodológicas durante el proceso de enseñanza aprendizaje en este nivel educativo en la Universidad.

Se constató que en total fueron ocho docentes, con una formación académica diversa en el nivel de licenciatura. De este total, dos se graduaron en universidades del Pacífico, mientras que los otros seis en la URACCAN, recinto Bilwi. Posterior a su formación general, el personal docente ha continuado estudios superiores, por lo que en la actualidad tienen el grado de maestría en Antropología Social y otros en docencia universitaria.

La URACCAN, como universidad comunitaria siempre está velando por la eficiencia de los procesos educativos y para tal fin continuamente está capacitando a su personal, siempre en busca de la calidad educativa. No obstante, en referencia a la temática de interculturalidad la coordinadora saliente del área de Humanidades expresó su percepción al respecto: "Me parece que muy pocos docentes aprovechan las capacitaciones, muestran poco interés, siempre quedamos con muchas lagunas, ¿Por qué?"

La misma informante amplió: "Nuestras capacitaciones han sido sólo conceptualizar y dar algunos ejemplos, pero no nos hemos metido a estudiar de manera profunda los fundamentos teóricos de la interculturalidad" (Entrevista a Davis, mayo 2010).

De alguna manera se refleja la resistencia a este tema, por ejemplo: "Algunos docentes dicen: ¿Cómo puedo aplicar interculturalidad en matemáticas? Es decir que todavía hay resistencia por algunos o algunas docentes para asumir esta temática como un eje transversal que debe estar presente en todo la labor educativa" (Entrevista a Davis, mayo 2010).

La expresión anterior lo remarca al decir: "en lo personal he sentido que todas las capacitaciones lo ven como algo folklórico, muy folklórico".

Aquí se comparte lo expresado por Velásquez (2002:99) y Krain (1994:44), quienes coinciden en que toda enseñanza de calidad requiere que se tenga claridad acerca de lo que se va a enseñar, además que se identifique con el quehacer pedagógico.

La secretaria académica del recinto, de alguna manera no concuerda con lo anteriormente citado porque su percepción es que hay mucha sensibilidad para atender los 
grupos de clase de manera diferenciada. Se hace visible la presencia de cada etnia con sus propios rasgos como lengua y cultura, los cuales tienen la misma importancia. Es decir, se ha despertado mayor interés en la articulación entre lo endógeno y lo exógeno.

Indiscutiblemente el personal docente en su misión como facilitadores de procesos educativos demanda de mucho estudio, pero también de apertura de pensamientos y esfuerzos para crear o recrear situaciones y actividades con el fin de promover aprendizajes significativos.

Además de la diversidad académica, existe la diversidad étnica, pues se encontró que la identidad de los docentes que facilitaron procesos educativos en el primer año corresponde a: una docente creole, dos mestizas, tres miskitus. Aunque no se encontró a nadie de la etnia sumo mayangna. En términos de género: cinco son mujeres y tres son varones, de tal manera que predominan las mujeres docentes.

Esta diversidad es un elemento enriquecedor, integrador y articulador, porque no trata de quedarse únicamente en las diferencias, sino que a partir de lo particular de cada una de las culturas, busca los puntos de coincidencia para fomentar la convivencia armónica, y, por ende, flexibiliza a que los procesos educativos sean pertinentes.

Obviamente, la diversidad étnica del personal docente ha contribuido en el cual el empleo del bilingüismo sea una estrategia metodológica implementada durante el proceso de enseñanza aprendizaje, es decir, que momentáneamente se auxilian de la lengua materna miskitu o hacen traducciones, lo cual no es un pecado, pero se incurre en algunas dificultades que provocan malestares en la comunidad estudiantil cuya lengua materna no es el miskitu, es decir, que tanto los creoles, mayangnas y mestizos se sienten al margen de lo que sucede en el aula de clases.

\section{Estrategias metodológicas y el desarrollo de la interculturalidad}

La diversidad étnica en clases, tanto de docentes como de estudiantes, es un reto para la universidad. Su perfil es comunitario e intenta desarrollar prácticas interculturales durante el proceso educativo, con el fin de lograr la convivencia armónica entre estudiantes. Sin embargo, la interrogante es ¿cómo implementarlo de manera permanente en la práctica educativa que se desarrolla en el aula de clases?

$\mathrm{Al}$ respecto la secretaria académica se refiere a los docentes en el sentido que elevan la autoestima de los estudiantes, aunque se dan casos en que ocurre todo lo contrario. Asimismo, expresa que desde la Universidad se trata de desarrollar procesos de reflexión sobre la importancia de la diversidad, de la diferencia que existe en este contexto, y cómo eso en vez de ser un problema más bien enriquece. 
Prosigue explicando que dichas acciones de docentes han contribuido a que: "se combata la burla y el irrespeto, es decir que se propician más los ambientes de confianza para expresar opiniones" (Entrevista a García, mayo 2010), aunque considera que hay momentos en que se cae en debilidades como el paternalismo, maternalismo y asistencialismo, confundiéndolo con acciones afirmativas. También se cuida el uso de palabras o chistes racistas, se dialoga más.

Obviamente, la práctica de la interculturalidad no es solamente la promoción del respeto y la tolerancia, sino que más bien busca incentivar el diálogo y el reconocimiento mutuo. Esto concuerda con lo señalado en el Modelo pedagógico de la URACCAN, en que la interculturalidad es un proceso donde se va construyendo un diálogo de iguales, donde las culturas pueden respetarse y crear juntos nuevos conocimientos.

Mediante las observaciones realizadas a pie de aula se constató que los maestros y maestras, muestran apertura y una actitud positiva hacia la atención de la diversidad étnica que tiene presencia en clases; así mismo, la mayoría de los estudiantes expresaron que sus docentes les inculcan valores como el respeto. Empero, no se trata sólo de actitudes positivas sino que las acciones que realizan permitan hacer realidad la verdadera interculturalidad desde las aulas de clases donde los docentes juegan un papel importante, para lo cual se requiere preparación y claridad sobre el rol que desempeñan como guías y facilitadores de los procesos educativos.

En el currículo de la carrera aún no se encuentran estrategias diseñadas que permitan poner en práctica la interculturalidad, tal a como lo reafirmó la secretaria académica del recinto quien señaló que no es generalmente una práctica intencionada ni planificada, pues en los planes de estudio no se encuentran objetivos dirigidos a la práctica de la interculturalidad.

Ella misma considera que: "Sí, hay acciones específicas como el uso del bilingüismo o el de partir por ejemplo de experiencias de la comunidad estudiantil que es un buen avance, además que no existe una metodología concreta para implementar la interculturalidad" (Entrevista a García, mayo 2010).

Los estudiantes coinciden al expresar que lo hacen mediante la conformación de equipos de estudio o de trabajos en grupos, donde no existe distinción de etnias ni de género, aunque cada docente tiene sus normas, siempre hay respeto entre ambos. En este sentido, continuaron narrando los estudiantes: "Todos nuestros maestros y maestras no tienen actitudes racistas, ya que no hay distinción de etnias a la hora de pedir opiniones, comentarios" (Entrevista a estudiantes, mayo 2010).

Otros estudiantes coincidieron al expresar: "En el aula de clases existe bastante respeto en cuanto a la diversidad étnica y por ende lingüística, ya que es meritorio mencionar que algunos de nosotros no manejamos el español, entonces los docentes 
dan la oportunidad para que hablen en su propia lengua y muchas veces, algunos docentes imparten la clase auxiliándose hasta de tres lenguas" (Entrevista, 2010).

La secretaria académica también enfatizó respecto a las estrategias metodológicas utilizadas por docentes en el hecho de partir por ejemplo, de las experiencias de los estudiantes, "es un buen avance", reitera al explicar que no hay uso de metodología concreta para implementar la interculturalidad; antes bien, en algunos casos se aplica cada vez más el Modelo pedagógico referente a los criterios de docencia, aprendizaje y metodología dirigida a contextos como el nuestro: multicultural, multiétnico y plurilingüe.

La coordinadora anterior de la carrera de Sociología expresó: "Lo que hacen los docentes es promover temas como el respeto de las creencias, costumbres. Es decir, que hay trabajo que se hace aunque el docente no está claro del tema e importancia de la verdadera interculturalidad, de algún modo aprovecha los espacios para explicar que es importante que nos respetemos unos a otros, que sepamos que no somos iguales y que eso no es ningún pecado, pero también desde el primer año enseñamos que es importante el respeto de sus ideas, las formas de ver el mundo" (Entrevista a Davis, mayo 2010). Es decir que se empiezan a despertar inquietudes sobre la interculturalidad.

Los docentes revelaron que las maneras en la realización de las prácticas educativas para el desarrollo de la interculturalidad ha sido mediante la aceptación de las diferentes personas que pertenecen a las otras culturas, y la relación que debe existir para poder entenderse entre ellas.

Explicaron que a la hora que se van a realizar los ejercicios: "conformamos grupos mixtos entre las diferentes etnias para que puedan dialogar y compartir sus experiencias". Obviamente, esto no es interculturalidad, sino que implica únicamente a la multiculturalidad. Indiscutiblemente para esto debe existir voluntad, tanto de los docentes como de estudiantes, para suprimir esas barreras y aprovechar esos espacios para que cada una de las culturas se desarrolle de manera autónoma.

En este aspecto, la educación que se brinda en la URACCAN juega un papel importante para la definición de la identidad, porque es aquí donde se abren debates en torno a esta temática en algunas asignaturas como Historia de la Costa Caribe, Sociología Urbana, Sociología Rural; además, que se trata de construir una ciudadanía intercultural.

Un aspecto importante a mencionar es la referencia de la secretaria académica que: "en términos generales me parece que van por buen camino porque hay voluntad". Sin embargo, difieren los docentes en que: "es difícil ponerlo en práctica pedagógicamente porque los programas no tienen diseñadas metodologías para tal fin". Esto indica que coinciden con lo referido por la coordinadora anterior de que: "si no estás 
claro de lo que es la interculturalidad, tampoco podrías ponerlo en práctica durante el quehacer docente".

Indiscutiblemente, si el maestro y la maestra no están preparados y abiertos al cambio, no podrán facilitar procesos educativos pertinentes que conlleven al logro de objetivos propuestos.

Se han brindado capacitaciones relativas a la conceptualización y apropiación de la interculturalidad, así como su aplicación en las clases, ante lo cual la secretaria académica del recinto reconoce que se han generado cambios, por ejemplo: "me parece que hay mayor sensibilidad para atender los grupos de clase de manera diferenciada, así como utilizar el idioma materno en el aula", expresó:

Se hace visible que cada etnia, lengua y cultura tienen la misma importancia, se ha despertado mayor interés en la articulación entre lo endógeno y lo exógeno. Algo importante es que aunque no se entienda, se respeta. Además, queda claro que hay gente que entiende, habla y hace las cosas de manera diferente y no por eso son mejores o peores que otros, ya que solamente son diferentes.

Los estudiantes en su mayoría concuerdan con lo externado por la secretaria académica, los docentes y la coordinadora de la carrera en cuanto a que: "Durante el trabajo en clases podemos expresarnos en nuestro propio idioma sin distinción de raza. Casi siempre se están utilizando las mismas estrategias, pero a nuestro criterio, las que más funcionan y contribuyen son los trabajos en grupos" (Entrevista a estudiantes, mayo, 2010).

Los planteamientos remiten a que se reflexione sobre la aplicación de las estrategias, las cuales juegan un papel importante porque sirven para generar aprendizajes a través de procedimientos, así como de las habilidades que los estudiantes adquieren y utilizan ante diversas situaciones que se les presente.

La coordinadora saliente del área de humanidades, también indicó: "me parece que hay asignaturas para hablar del tema de interculturalidad como la historia, donde se explica la conformación de diferentes pueblos, tratan de explicar el por qué de las jerarquías sociales y etnolingüísticas que todavía persisten". Además que, "en otras, siento que los docentes llegan completos (o sea con el tiempo limitado) a dar su clase, mientras que la interculturalidad es algo aparte que sólo le corresponde a algunos docentes". Aquí se deja claro que no toda la planta docente asume el compromiso con el quehacer educativo de la Universidad. Esto se debe en parte a que algunos docentes son contratados para desarrollar un programa de clases y luego se retiran" (Entrevista Davis, mayo, 2010). 
Los docentes al ser entrevistados manifestaron que: "a nivel de aula comparten sus conocimientos e inciden a través de sus ejemplos, para que luego lo practiquen con sus compañeros, compañeras, en familia, vecinos u otras personas, por medio del diálogo, demostrando respeto hacia el otro u otra".

\section{Rol estudiantil}

En el proceso investigativo se detectó que existen dificultades en relación a las prácticas interculturales en clases de primer año, porque al llegar estos estudiantes por primera vez a la Universidad, además de ingresar a una nueva modalidad y al nivel superior de educación, también coincide el encontrarse entre diversas comunidades y colegios. Esta situación muchas veces hace que se presenten malos entendidos entre ellos, así comoburlas, muchas veces hasta pleitos donde tiene que intervenir la coordinación de la carrera.

De igual manera, "al inicio hay poca confianza, pero lo logran superar pronto con el apoyo y orientación de los maestros y maestras. Aunque en general se pueden calificar como cordiales, respetuosos entre ellos". En este sentido la educación juega un papel destacado para la configuración equilibrada y ajustada de las identidades culturales. Tal a como plantea Ramírez (2002) sobre algunas actitudes que deben conformar la competencia intercultural, entre las cuales menciona la "capacidad de resolución pacífica de conflictos", así como la "capacidad para trabajar en equipos persiguiendo metas compartidas".

En el caso específico de la carrera de Sociología con mención en Autonomía se pretende que el estudiantado adquiera formación en la promotoción y valoración de los saberes ancestrales. Estudiantes con orgullo de la pertinencia étnica, con capacidad de incidir en políticas locales, regionales y nacionales. Por lo tanto, la Universidad tiene bajo su responsabilidad el diseño de estrategias didácticas para favorecer las relaciones interpersonales y el conocimiento mutuo entre las culturas.

\section{Incidencias de las estrategias metodológicas para el desarrollo de la interculturalidad}

La formación académica del personal docente en la URACCAN, recinto Bilwi, ha incidido en el empleo de estrategias metodológicas durante el proceso de las diversas clases. Sin embargo, aunque se tienen incidencias positivas, se siguen encontrando algunas limitaciones que no permiten el desarrollo y avance de las prácticas interculturales en clases.

Prevalece el uso del bilingüismo como estrategia que permite la participación, es decir, que hay momento que se auxilian de la lengua materna miskitu o traducen según se requiera, lo cual ocasiona algunas dificultades que a su vez provocan malestares 
en estudiantes cuya lengua materna no es el miskitu; es decir, que tanto en creoles, mayangnas y mestizos hay ciertas susceptibilidades en el proceso educativo de ampliar sus mentes.

En referencia al currículo de la carrera de Sociología con mención en Autonomía, en cuanto a los programas que se aplican en el primer año, los docentes manifestaron que sí permiten las prácticas interculturales durante el proceso de enseñanza aprendizaje, esto porque: "forman parte de la realidad de esta área del país". Empero, la estructura que tienen los programas es generalizada, no muy coherentes para una universidad comunitaria e intercultural.

Lo expresado sobre estrategias de enseñanza, conlleva a reflexionar en que dicho término supera académica y pedagógicamente al de la metodología, debido a que constituye un nivel de complementariedad y síntesis de todos los procesos didácticos educativos.

Se considera como fortalezas lo expresado por la secretaria académica del recinto, en cuanto a que algunos docentes promuevan en la comunidad estudiantil el que se hable en su lengua materna. Esto hace que la gente se sienta orgullosa de sí misma, que la interculturalidad pase a apropiarlo de lo suyo y se respete la del otro. También el uso del bilingüismo por docentes y estudiantes, es decir el uso de la lengua materna y del español como segunda lengua.

La formación de grupos de estudio o trabajos durante el proceso educativo es el elemento en que coinciden las fuentes investigadas para este trabajo de tesis.

Asimismo, se presentan algunas limitaciones al no ponerse de acuerdo el personal docente sobre el tratamiento metodológico que se dará en clases para atender la diversidad étnica y cultural, mediante estrategias metodológicas que promuevan las practicas interculturales.

\section{Conclusiones}

En base a los resultados obtenidos en el presente trabajo investigativo se presentan las siguientes conclusiones:

La estrategia metodológica que prevalece durante el proceso de enseñanza aprendizaje son los trabajos en grupos.

Las estrategias metodológicas deben diversificarse, de tal modo que ayude a relacionar los procesos de enseñanza y aprendizajes con la diversidad étnica y cultural. 
Aunque en la planta docente no todos estén claros completamente de la interculturalidad, de alguna manera se pueden aprovechar espacios para explicar que es importante que nos respetemos unos a otros.

Para vivir la multiculturalidad y la interculturalidad en el aula se debe tener como base la planificación curricular orientada desde los principios de la multiculturalidad y la interculturalidad.

Las experiencias académicas interculturales deben abarcar todos sus componentes como el currículo, la metodología, la participación de los distintos agentes educativos.

La interculturalidad, además de favorecer el respeto entre las culturas, busca la interacción entre culturas sin perder la propia.

En la carrera de Sociología con mención en Autonomía por su perfil permite aplicar el enfoque de interculturalidad desde los contenidos programáticos de algunas asignaturas, pero se trata de aplicar más en la relación estudiantes y docentes, en el fomento de actitudes de respeto a la diversidad étnica.

La función docente requiere de varias herramientas para alcanzar sus propósitos. Por tanto, deben poner en juego toda su creatividad para lograr que sus estudiantes se formen y dominen los conocimientos, las actitudes y desarrollen las habilidades deseadas, para convivir e interactuar dentro de los procesos educativos.

\section{Lista de referencia}

Arroyo, Rosario (1998). La escuela y el currículo intercultural. Perú -

Batista, Ángel (2000). Procesos, técnicas y estrategias didácticas para enseñara aprender a aprender. ISAE. Veraguas.

Bolívar, Burbano Paredes (1994). Docencia Bilingüe Intercultural. Una especialización para nuestros días. Edic. Abya Yala. Quito Ecuador.

Bustamante, Marta Elizabeth (1996). Pedagogía y formación docente. $1^{\circ}$. Edición, Cartago, Costa Rica

Díaz, Frida (2005). Estrategias docentes para un aprendizaje significativo.

Giménez, C. (2000) Guía sobre interculturalidad. Fundamentosconceptuales. Colección cuadernos Q'anil 1, Guatemala, Proyectos Q'anil B.

Heise, María (1999). La interculturalidad, un desafío. Lima, Perú. 
Kraine Anita (200o). Educación Intercultural Bilingüe. $1^{0}$ edición.

MINED. (2006). Aportes de la psicología al aprendizaje de los niños y niñas y adolescentes.

McWilliam, Norah (1990). Didáctica general.

Pineda Moctezuma Angélica (1993). Evaluación del Aprendizaje. Editorial Trillas, México.

PNUD. (2005). Informe de Desarrollo Humano 2005. Las Regiones Autónomas de la Costa Caribe. Nicaragua asume su diversidad. Managua.

Ramírez Vargas, Margarita (2002). Tradición oral en el aula.

Rizo, Mario. (2003) Cultura. Materiales de apoyo para la formación docente en educación intercultural. Managua. IPILC URACCAN con el auspicio de IBIS, KEPA y Terra Nuova a través del proyecto SAHWANG.

Saavedra, (1996) Primer encuentro nacional salud y pueblos indígenas. Región de la Araucanía, Chile.

Saballos Velásquez, José Luis (2007). Módulo 1. Construyendo conceptos. ${ }^{\text {a }}$. Ed. Managua: URACCAN.

Sola Mendoza, Juan (1998). Pedagogía en Píldora. Editorial Trillas, México.

Us Soc, Pedro (2002). La práctica de la interculturalidad en el aula. Primera edición. Cartago, Costa Rica.

UCA ASEN (S/f), Marco global y nacional de la Educación - Reforma educativa (compilación). Managua, Nicaragua.

URACCAN (2004). Modelo pedagógico.

URACCAN (2000). Sistema Educativo Autonómico Regional SEAR $1^{\circ}$. Edición.

Velásquez de Bustamante, Martha Elizabeth (2002) Pedagogía y formación docente. Primera edición, Cartago Costa Rica,

Venezia Mauceri Paolo (2003) Educación intercultural y plurilingüe. Primera edición. Managua. TerraNuova.

Vigil, N y Zariquiey, R. (2003). Ciudadanías inconclusas. El ejercicio de los derechos en sociedades asimétricas. Lima, Perú Pontificia Universidad Católica del Perú con el auspicio de GTZ. 